\title{
Internet technology: contributing to agility in the twenty-first century
}

\author{
Ronald E. McGaughey \\ Arkansas Tech University, Russellville, Arizona, USA
}

\begin{abstract}
Keywords
Agile production,

Computer technology, Internet, Organizational change
\end{abstract}

\section{Abstract}

To compete effectively in many developed and emerging global markets firms must develop the capacity to react quickly and successfully to change - they must become agile. Internet technology, in the form of corporate intranets and the Internet, can be an important enabler of agility. Internet technology makes possible the intra- and inter-organizational sharing of data and information in the form of text, graphics, audio, and video, enabling various tasks, activities and processes that help a firm to become agile and ready to compete in the twenty-first century. This manuscript examines Internet technology's contributions to agility.

International Journal of Agile Management Systems

1/1 [1999] 7-13

C MCB University Press [ISSN 1465-4652]

\section{Introduction}

Agility as the term is used herein is the ability of an enterprise to respond quickly and successfully to change. For a company to be agile it must be capable of operating profitably in a competitive environment of continuous and unpredictable change (Goldman et al., 1995). Why profit from change? Because change is a certainty for the twentyfirst century.

Since 1991, when the term agile was first used in describing a capability needed in modern manufacturing (Litsikas, 1997), most of the research on and discussion of agility has centered on manufacturing. Agile manufacturing is now sharing press with the broader concept of an "interprise," which is any type of enterprise striving to become agile - develop the capabilities and competencies that will be needed for sustained business success in the twenty-first century (Preiss et al., 1996). Like manufacturers, retailers, wholesalers and pure services must become more agile to compete in the twentyfirst century.

There has been much discussion of the contributions of human systems and technical systems to agility. A knowledgeable and highly motivated workforce organized in a manner to facilitate rapid realignment in anticipation of or as a response to change is a critical ingredient in agility (Preiss et al., 1996). The technology employed by organizations must support the activities and tasks required in and between firms to continuously meet changing customer needs. One technical subsystem of particular importance to agility in our high-tech, connected world, is the telecommunications network.

A network is made up of hardware and software that link computers so that they can share data and processing responsibilities. More generally a network connects individuals, groups, organizations and machines thereby supporting intra- or inter-organizational data communications and fundamental business processes.

The Internet, a global "network of networks" (Carr and Snyder, 1997, p. 368), is, without question, the most important network in the world today. This loosely configured web of corporate, educational and research computer networks (Tetzeli, 1992), driven by its graphical user interface, "the Web," has reshaped the very nature of commerce, entertainment, and human connectivity (Metes et al., 1998). The popularity of the Internet spawned a new type of corporate network called an Intranet.

An intranet is in essence a "private Internet" (Christensen, 1996). Intranets, which have become enormously popular, make use of extremely popular and low cost Internet tools. From a user's perspective an intranet looks like the Internet and acts like the Internet. This is why many people, particularly younger employees, can quickly adapt to intranet use. While an intranet is more limited in scope (there may be less to see and use), it is typically faster, more secure and more reliable than the Internet (Christensen, 1996). These intranet advantages benefit users and the company.

Intranets and the Internet use the same TCP/IP network protocol, and E-mail and world wide web (WWW) standards (Christensen, 1996). Both the Internet and intranets make possible the sharing of data and information in the form of text, graphics, audio, and video, thereby supporting a wide range of individual and group processes that once required on-site, face-to-face communications. Internet technology is the common technological foundation for Intranets and the Internet.

In a book entitled Agile Networking: Competing through the Internet and Intranets, Metes et al. (1998) examine the role of networking in implementing agile processes and use the term "agile networking" to describe the union of agility and networking. They assert that networking provides one of the "hows" for agility. Like these authors, I am convinced that Internet technology can play a prominent role in helping firms to become more agile. I believe that intranets and the Internet provide numerous "hows" for agility. In the following pages I will examine some Internet and intranet capabilities that can contribute to agility now and in the twenty-first century. 
Ronald E. McGaughey Internet technology:

contributing to agility in the twenty-first century

International Journal of Agile

Management Systems

1/1 [1999] 7-13
The strategic dimensions of agility: a useful framework

Goldman et al. (1995) identified four strategic dimensions of agile manufacturing which are applicable, also, to non-manufacturing enterprises. They are as follows:

(1) enriching the customer,

(2) cooperating to enhance competitiveness,

(3) organizing to master change and uncertainty, and

(4) leveraging the impact of people and information.

The Agile Manufacturing Research Institute uses these strategic dimensions in considering the tactical and technological dimensions of agility (DeVor et al., 1997). These dimensions will provide a useful context for examining how Internet technology (intranets and the Internet) contributes to agility.

\section{Contributions to customer enrichment}

Enriching the customer involves supporting the customer's processes in a way that is perceived by the customer to be enriching. Rusty Patterson, Industry President and CEO of the Agility Forum of Bethlehem, PA describes this as "giving the customer what he wants throughout changing needs and throughout the life of the product" so that "he won't have to go anywhere else" (Litsikas, 1997, p. 31). By enriching its customers an enterprise can become a permanent part of its customers' processes.

One very important role Internet technology can play in enriching customers is in monitoring ever-changing customer needs. A better understanding of customers and their needs is a prerequisite for meeting changing customer needs on an ongoing basis. When Internet technology (Internet and/or intranet) is used to support transactions, organizations have an opportunity to capture, electronically, data on what and how much customers purchase, timing and frequency of purchases, how customers pay and how much they pay. Basic data on customer demographics are typically available as a part of the transaction and other data might be solicited from customers during transactions.

The Internet offers considerable potential in collecting data on customers when transactions are not completed online, or to supplement data collected from online transactions. Companies can use the Internet to stay abreast of consumer needs by establishing and maintaining an ongoing dialog with customers through the use of E-mail or chat rooms. An E-mail-based dialog between company personnel and customers might be used to solicit feedback about products, monitor customer needs, and follow up on customer purchases providing further assistance if warranted. E-mail surveys can be used to collect data on customer needs.

A company supported Web page can aid in the collection of data relating to customer needs and more. If an enterprise can get customers or prospective customers to visit its web page, it can use various Web-based tools to solicit feedback about the depth and breadth of its product offering and the quality of its products, and to solicit customer ideas for product modifications or new products. Web pages provide a useful vehicle for collecting a wide variety of data about customer needs and for involving customers in decisions that concern them.

The Internet now makes it possible for consumers to experience certain types of products or product characteristics after which a company might solicit feedback about consumers' experiences. Trial versions for some products - software demos are a good example - are deliverable (downloadable) by Internet. Though not commonplace at present, customers, or potential customers will be able to experience products, or product features, through an Internet-based virtual reality experience. User responses to inquiries regarding experiences with product demos, or virtual reality experiences, could provide meaningful insight into present and future customer needs that would be helpful in tailoring products to customer needs.

Software providers are using the Internet creatively to enrich their customers by creating installation programs that allow users to opt for receipt of automatic notification and/or installation of software updates. In the service sector banks are enriching customers by using telecommunications technology to support an increasing number of tasks and activities that comprise the customer's financial management process. Secure networks allow customers to access information on account balances, transfer funds, make deposits, pay bills, buy and sell stocks, submit loan applications and more without the direct assistance of bank personnel. Internet technology allows banks and other financial institutions, such as credit unions, to vastly expand their role in the customer's financial management process.

For manufacturers the Internet is a vehicle for involving customers in product design processes. Ford Motor Company used online conferences and E-mail to support customer interaction with designers working on the 
Ronald E. McGaughey Internet technology:

contributing to agility in the

twenty-first century

International Journal of Agile

Management Systems

1/1 [1999] 7-13
Taurus automobile (Preiss et al., 1996). This helped Ford to get customers more involved in the product design process. Ford management believes that the program will produce a tighter coupling of company and customer processes and greater customer loyalty.

Other developments in the automobile industry offer great promise in securing manufacturers a more prominent role in ongoing automobile maintenance. On-board computers make it possible for automobile companies to send E-mail to owners in their automobiles reminding them when maintenance is due, recommending nearby service centers, quoting prices for the maintenance, and scheduling maintenance appointments. On-board global positioning systems can direct customers to service centers.

Web-based customer service offers potential for enriching customers. Using pictures, video, and audio, company personnel can literally show a customer a product, how to use a product or solve a problem, and explain things in much the same way as would be done in face-to-face interaction with the customer. A "chat room" type capability can allow customers to carry on a dialog with a company representative to get help with a product, or get information on a product or service. Employees addressing a customer problem interactively can use the company's intranet to get help from other employees anywhere in the company who might have the necessary knowledge to be of assistance, or use the Internet to take advantage of the expertise of personnel in other organizations.

There are many other ways in which the Internet, or an intranet might assist organizations in their efforts to enrich customers. The limits of Internet technology support for the enrichment of customers seem at present to be due more to the limits of human imagination than the limits of technology.

\section{Facilitating cooperation to enhance competitiveness}

Cooperation is a cornerstone of agility. It is an important philosophical underpinning for agility (Nagel and Dove, 1993). Cooperation is necessary within an organization as a means of synchronizing the many people and organizational subunits that play a role in bringing about the actions required to continually meet ever-changing customer needs. The need for cooperation extends beyond the firm to customers, suppliers, stockholders, government, and even competitors. Team membership can be extended to any organization, or entity that can help an enterprise become and remain a valuable enabler in the customer's process.

Inter- and intra-organizational cooperation requires the interaction of individuals and groups. Verbal or non-verbal communications is the primary driver of meaningful human interaction in and between organizations and, thus, communications is the key to cooperation. It is the role of Internet technology in facilitating communications that makes it important in bringing about cooperation. Preiss et al. (1996, p. 86) have called the Internet a "universal medium for business interaction." Companies are using intranet capabilities to enhance intra-company communications and the Internet to support interaction with the rest of the world.

Much of the interest in the Internet technology stems from its capacity to make information available to people in the form of text, graphics, audio and video (Gundry and Metes, 1997). The intranet supports the sharing of corporate data across departmental barriers and among geographically dispersed individuals and groups. The Internet makes possible the sharing of data or information among people, groups and organizations all over the globe. The underlying infrastructure that supports the Internet and intranets allows data to be provided to those that need them, when they need them, and in a form most useful to them. Since intranets and the Internet are based on a common infrastructure, data can be simultaneously shared within and outside an organization. E-mail, for instance can be sent to targeted people within and outside an organization. Web page contents can be shared within an organization and with others across the globe. Audio and video conferencing (desktop or studio), as well as groupware products like Lotus Notes, can involve people from various areas of an organization and people from other organizations in collaborative work.

As an illustration of how an intranet can facilitate cooperation among individuals or groups in an organization, take the example of a modification to a product design in response to changing customer needs. An intranet-based groupware product could facilitate the sharing of information regarding customer needs and collective interpretation of those needs, and it could support collaborative work on product and process design. A sales person familiar with the customer, a product engineer, a process engineer, a purchasing manager, a production/service manager, and a worker (or workers) responsible for making the product/providing the service could all provide input into the product design and simultaneously for process design. Participants could share data in 
Ronald E. McGaughey Internet technology:

contributing to agility in the

twenty-first century

International Journal of Agile

Management Systems

$1 / 1$ [1999] 7-13 an appropriate format to convey the relevant details concerning customer needs and the product or process design (a drawing, comments in text or audio, and video if warranted). Such a capability could make it possible for anyone, anywhere in the organization with intranet access, to participate in the process of designing products and processes to better meet customer needs.

The above illustration focused on intranet support for participation of individuals and groups within an organization. Internet support would allow an organization to involve participants from other organizations in the process. The customer, suppliers, consultants or virtually anyone with a stake in the process could be involved in the process. Internet support, providing some of the same capabilities discussed above, allows an organization to extend collaborative work beyond organizational boundaries.

The push for agility has, in some instances, resulted in what has been called a "virtual business". A virtual business is a seamless and borderless web of companies, which have linked themselves for the purpose of taking advantage of business opportunities beyond the reach of any one firm acting alone. The virtual enterprise can take advantage of business opportunities greater than the sum of the contributors' capabilities (Preiss et al., 1996). The virtual business involves intensive, interactive relationships among participants that require sharing information within and among companies. Business systems and processes must be totally integrated, yet organizational relationships are dynamic and membership changes over time. It is not practical to develop an entirely new information infrastructure every time membership changes, so Internet technology is an appropriate telecommunications infrastructure for supporting the virtual business. As virtual business relationships change, Internet technology will allow former participants to be "unplugged" and new participants to be "plugged in."

Cooperation requires communications. In an agile enterprise, some cooperative arrangements may be stable, but others change as relationships and teams evolve to address the challenges of a changing world. The dynamic inter- and intra-organizational cooperation necessary for agility require a communications infrastructure that can support both stable and evolving relationships. Internet technology provides that infrastructure.

\section{Contributions in organizing to master change and uncertainty}

Flexibility is the key! As noted, an agile enterprise must be appropriately organized to thrive on change and uncertainty (DeVor et al., 1997). As any part of the firm's environment changes, it must be sufficiently flexible to reorganize its human systems and technical systems to not just adapt to change, but to take advantage of change.

In the past the primary motive of most firms in adopting new communications technology has been lower cost. Firms pursued what Sproull and Kiesler (1991) called the "first level" effect of communications technology - improvements in efficiency and productivity which lower cost. While low cost is important to firms striving to become agile, the second level effect of communications technology may be of greater importance in bringing about agility. Sproull and Kiesler (1991) asserted that the second level effect of communications technology is the way it changes patterns of interaction, work processes, and social organization (Sproull and Kiesler, 1991). This second level effect is of particular importance to firms striving for agility because of the impact on organizational structures and processes. Internet technology offers benefits pertaining to the first level effect of communications technology but, more importantly, it has the potential to produce a significant second level effect.

Intranets facilitate interpersonal communications and can encourage new patterns of relationships within organizations (Gundry and Metes, 1997). The Internet extends the secondary effect to communications and relationships with members of other organizations. Both the Internet and intranets provide rich communication facilities using common protocols across software, platforms and networks. Even as the Internet/intranet standards and protocols evolve, the underlying telecommunications infrastructure provides stable, yet flexible support for dynamic intra- and inter-organizational relationships.

Organizations using intranets and the Internet can reorganize and reconfigure human systems and technical systems as needed to take advantage of change without the need to make major changes in the underlying telecommunications infrastructure that links human systems (individuals, groups and organizations) and technical systems (machines, equipment and computer systems). New patterns of communication, relationships and workgroups can evolve quite naturally to solve problems, or take 
Ronald E. McGaughey Internet technology:

contributing to agility in the

twenty-first century

International Journal of Agile

Management Systems

1/1 [1999] 7-13 advantage of opportunities without restrictions imposed by time, distance and incompatible infrastructures. Political issues such as who owns the data do not distract members from their tasks, because intranets make it possible for those who need the data to access the data.

Internet technology allows knowledge workers to form, change and dismantle virtual intra- or inter-organizational teams in response to changes in any element of the organization's environment. This is precisely the capability firms must possess if they are to become sufficiently agile to compete in the twenty-first century.

\section{Leveraging the impact of people and information}

It takes more than advanced technology to achieve agility. Agility requires an entrepreneurial company culture that "leverages the impact of people and information on operations" (DeVor et al., 1997, p. 814). The entrepreneurial culture required for agility should encourage creativity, the free flow of information and exchange of ideas, cooperation, collaborative intra- and inter-organizational work, individual initiative and personal responsibility. It is a culture in which people and groups must be empowered to act in response to present and future customer needs.

Fundamental changes in organizational culture and structure are often necessary in firms striving for agility. In most cases, organizational structures which focused attention on hierarchy, rigid reporting relationships, division of labor, and accountability must be abandoned and replaced by a new corporate architecture that emphasizes results. Dess et al. (1995) fittingly described this new corporate architecture as boundaryless. Their notion of a boundaryless organization is quite consistent with the requirements for agility. Agile organizations should be boundaryless - they should not be stifled by rigid internal and external boundaries and structures that restrict information flows, limit flexibility, and promote mindsets and behaviors incompatible with agility.

The fundamental changes is business processes and practices which bring about agility are driven by human decision makers. These human decision makers must be delegated the authority to make decisions, have an entrepreneurial mindset (fostered by the proper organizational culture), be knowledgeable, and have ready access to the information they need to make sound decisions.
The importance of information in empowering people can hardly be overstated.

Authority to act is not sufficient to lead to good decisions. People need knowledge and information. The empowerment of individuals and groups requires authority, knowledge, and access to information. Internet technology leverages the value of information because it spans internal and external organizational boundaries making it possible to provide the information that is needed, to whomsoever requires it, when it is needed, in a form appropriate for use. Internet technology provides a pipeline for data flows to and from practically any human, machine, internal or external source (management, factory floor, store, warehouse, engineering, accounting, customers, suppliers, consultants, government(s), competitors, colleges and universities). This infrastructure enhances an organization's ability to educate and train and to provide accurate, timely data and information in a form suitable for internal and external constituents.

Education and training are very important in leveraging the skills and knowledge of people. Internet technology can expand education and training opportunities both within and among organizations because it reduces or removes many barriers such as cost, distance and inconvenience that have limited availability in the past. Web-based education and training is a rapidly growing phenomenon. Universities are using the Internet to deliver courses online and in some cases it is possible to complete an entire degree program online. Organizations are experimenting with the use of intranets and the Internet in employee education and training. The variety of information forms and formats possible with Internet technology allows organizations to deliver education and training to employees or other constituents all over the globe. Through the creative use of audio, video, graphics, and text, the experience of students or trainees can be much the same as if they were participating in activities and processes on site.

In a very basic way the Internet empowers people because it literally places a world of information at their fingertips. The Web (World Wide Web) allows workers with the URL of the target site, or with the help of search engines, to get information on just about anything. Information on products, processes, suppliers, competitors, consultants, and government regulations can be found in minutes, or even seconds by someone with a basic knowledge of the Web and Web tools such as the search engine.

Intranets can selectively provide individuals and groups, within or outside an 
Ronald E. McGaughey Internet technology:

contributing to agility in the

twenty-first century

International Journal of Agile

Management Systems

1/1 [1999] 7-13 organization, access to corporate data. The entire corporate database can be made available via an intranet. This is not to say that every person has access to all data. Levels of security can be implemented to limit access to sensitive data such as trade secrets, certain financial data, personnel files and such. Those individuals or groups who need access to specific corporate data can have access for purposes of monitoring, analyzing, and updating the data.

E-mail allows people to share information with individuals or groups inside or outside the organization. Even with E-mail sent across the globe, response times in seconds, minutes and hours are possible where conventional "snail" mail may take days or weeks and telephone tag can go on for days. Of particular importance with newer E-mail systems is the capability to transfer data/ information in a usable form. The ability to attach objects to E-mail makes it possible, and easy, to send the information in any number of forms or formats that would be suitable for the recipient. Video and audio clips, graphic images (such as a design produced by a CAD system), word-processing documents, spreadsheets, and database tables are among the many forms and formats for information that can be attached and sent as E-mail. E-mail makes it quick and easy to send and receive data and information in a useful electronic format thereby providing an efficient alternative to traditional mail and telephony. E-mail may in some cases be a viable alternative to electronic data interchange (EDI) and other specialized communication technologies.

Information should be made available to those that need it to make decisions and it should be provided in a timely fashion. The intranet facilitates timely access to data and information because time and distance do not limit access. One particularly important advantage of a corporate database (centralized or distributed) shared via an Intranet, is that there can be a single source for current data and all organizational personnel can be provided access to the data they need to do their jobs. Personnel can base decisions on accurate and timely facts and figures rather than their own versions of the facts and figures. Database contents can be supplemented with information from other sources to create a more complete and accurate context for decisions. Web pages can be created and maintained to supplement the corporate database by providing additional details about corporate strategy, business plans, programs and projects, planning premises, policies, team projects and performance, meetings, agendas, suppliers, customers and just about anything else. Email can be used to make inquiries about data when further clarification is desired by intranet users.

Internet technology can assist organizations in providing workers and groups inside or outside the organization with access to the data and information they need to make sound decisions in response to, or in anticipation of, change. Intranets and the Internet provide important support for education and training and can contribute substantially to ongoing efforts to develop a more knowledgeable workforce. Knowledge is a key ingredient in sound decisions and new knowledge is essential to firms that wish to thrive in an environment of rapid change.

\section{Summary and conclusion}

Internet technology can assist firms in their efforts to achieve agility. Internet technology helps to enrich customers in a variety of ways from monitoring customer needs to better supporting customer processes. Internet technology provides the communications infrastructure that enables communications, in a variety of forms and formats, among individuals, groups and organizations to facilitate the intra- and inter-organizational cooperation necessary to achieve agility. Internet technology provides a flexible communications infrastructure that can support the constantly changing intra- and interorganizational relationships that are necessary for success in an environment of rapid change. Internet technology leverages the impact of people and information. The value of information is leveraged when it is available to those that need it, when they need it, where they need it and in a form useful to them. People are leveraged when they are empowered with the knowledge and information they need to make decisions and perform job-related tasks. Internet-technology provides the vehicle for delivering information and for delivery of education and training that expands the knowledge of workers, both of which help to empower individuals and groups to act in ways that contribute to agility.

I find no shortage of anecdotal evidence to support my assertions about the importance of the Internet and intranets in promoting agility, yet anecdotal evidence is of limited value in providing guidance for practitioners and researchers. Researchers are devoting significant time, effort and resources in an attempt to determine how enterprises of all types can become sufficiently agile to compete in dynamic global markets. On another 
Ronald E. McGaughey Internet technology:

contributing to agility in the

twenty-first century

International Journal of Agile

Management Systems

1/1 [1999] 7-13 front, researchers examine the Internet and intranets in an effort to understand their characteristics, capabilities and future directions.

Having devoted considerable time to examining the literature on agility and the literature on Internet technology it is my conclusion that firms are missing significant opportunities to use the Internet and Intranets in ways that would help them to become more agile. For whatever the reason, Internet technology is underutilized by many firms striving for agility. There is a need for studies that meld research on agility with research on Internet technology and provide practitioners with guidance on how to use Internet technology to become more agile. Those who are willing to undertake this challenging work could help firms prepare to compete successfully in the twenty-first century.

\section{References}

Carr, H.H. and Snyder, C.A. (1997), The Management of Telecommunications, Irwin, Chicago, IL.

Christensen, R. (1996), "Intranet: misspelling...or megatrend?" Reprinted by permission, Atlanta Computer Currents. Available from CTS Web Publishing and Document Conversion. <http://www.mindspring.com/ rchris/ ct00002.htm > access date 12/16/98.

Dess, B., Rasheed, M.A., McLaughlin, K. and Priem, R. (1995). "The new corporate archi- tecture," Academy of Management Executive, Vol. 9 No. 3, pp. 7-17.

DeVor, R., Graves, R. and Mills, J.J. (1997), “Agile manufacturing research: accomplishments and opportunities," IIE Transactions, Vol. 29, pp. 813-23.

Goldman, S., Nagel, R. and Preiss, K. (1995), Agile Competitors and Virtual Organizations, van Nostrand Reinhold, New York, NY.

Gundry, J. and Metes, G. (1997), "Internet challenges: online work and communication," copyright 1997 by Knowledge Ability Ltd and Virtual Learning Systems, Inc. <http:// www.knowab.co.uk/wbwintra.html $>$ access date $12 / 14 / 98$.

Litsikas, M. (1997), "Quality makes agile manufacturing possible,” Quality, Vol. 36 No. 2, pp. 30-8.

Metes, G., Gundry, J. and Bradish, P. (1998), Agile Networking: Competing through the Internet and Intranets, Prentice-Hall PTR, NJ.

Nagel, R. and Dove, R. (1993), 21st Century Manufacturing Enterprise Strategy, Iacocca Institute, Lehigh University, Bethlehem, PA.

Preiss, K., Goldman, S. L. and Nagel, R.N. (1996), Cooperate to Compete: Building Agile Business Relationships, van Nostrand Reinhold, New York, NY.

Sproull, L. and Kiesler, S. (1991), Connections: New Ways for Working in the Networked Organization, The MIT Press, Cambridge, MA.

Tetzeli, R. (1992), "The Internet and your business”, Fortune, March 7, pp. 86-96. 